INPLASY

PROTOCOL

To cite: Li et al. A protocol for systematic review and network meta-analysis Efficacy and Safety of Kunxian Capsule in Treating Patients with Lupus Nephritis: A network metaanalysis. Inplasy protocol 2021120005. doi:

10.37766/inplasy2021.12.0005

Received: 01 December 2021

Published: 01 December 2021

Corresponding author:

Zifeng Li

1002581008@qq.com

Author Affiliation:

Department of traditional

Chinese Medicine, The First

College of Clinical Medical

Science, Three Gorges

University/Yichang Central

People's Hospital.

Support: None.

Review Stage at time of this submission: The review has not yet started.

Conflicts of interest:

None declared.

\section{A protocol for systematic review and network meta-analysis Efficacy and Safety of Kunxian Capsule in Treating Patients with Lupus Nephritis: A network meta-analysis}

Li, Z1; Fan, Y2; Lei, Y3; Hou, X4; Chang, Y5.

Review question / Objective: To study on the efficacy and safety of Kunxian Capsule in treating patients with lupus nephritis( LN).

Condition being studied: The study showed that Kunxian capsule significantly improved immune status and renal function and suppressed IL-10 and TNF in serum of lupus mice- a Of secretion, works better in combination with and reduces hormone dosage. At present, there are few studies in which Kunxian capsules are used in the clinic, and the conclusions of the studies are inconsistent, so this study was conducted in order to comprehensively evaluate the efficacy and safety of Kunxian capsules for the treatment of lupus nephritis.

INPLASY registration number: This protocol was registered with the International Platform of Registered Systematic Review and Meta-Analysis Protocols (INPLASY) on 01 December 2021 and was last updated on 01 December 2021 (registration number INPLASY2021120005).

\section{INTRODUCTION}

Review question / Objective: To study on the efficacy and safety of Kunxian Capsule in treating patients with lupus nephritis( LN).
Condition being studied: The study showed that Kunxian capsule significantly improved immune status and renal function and suppressed IL-10 and TNF in serum of lupus mice- a Of secretion, works better in combination with and reduces hormone dosage. At present, there are few studies in 
which Kunxian capsules are used in the clinic, and the conclusions of the studies are inconsistent, so this study was conducted in order to comprehensively evaluate the efficacy and safety of Kunxian capsules for the treatment of lupus nephritis.

\section{METHODS}

Search strategy: Six electronic databases (Web of Science, PubMed Database, Embase Database, the Cochrane Central Register of Controlled Trials, China National Knowledge Infrastructure, Wanfang database and the Chongqing VIP Chinese Science) will be searched from May 1st, 2011 to May 1st, 2022.

Participant or population: Patients with lupus nephritis will be included. Our review included studies using Kunxian Capsule. There will be no restrictions in terms of the patients age, gender, race, education or economic status.

Intervention: All treatments containing Kunxian capsules(Treatment duration was 12 weeks).

Comparator: Do not make restrictions.

Study designs to be included: Intervention studies or observational studies.

Eligibility criteria: Inclusion and exclusion criteria are categorized by population, interventions, comparators, outcomes and study design (PICOS). The year of publication is restricted from 2011-2022 and there were no restrictions regarding the language of the article.

Information sources: Web of Science, Medline, Embase and Cochrane Central Register of Controlled Trials will be been comprehensive searched. Given that Kunxian Capsule is widely used in China, we will search the following Chinese databases: China National Knowledge Infrastructure, the Chongqing VIP Chinese Science and Wanfang Data from May 1st, 2011 to May 1st, 2022. The search strategy for PubMed is as follows (Table1). And the equivalent search words will be used in other databases as well.

Main outcome(s): SLEDAl scoring,24-hour urinary protein quantity.

Additional outcome(s): Creatinine (Cre), C3, C4, IgG, IgM, anti-mullerian hormone (AMH), Adverse event rates (Leukopenia, thrombocytopenia, respiratory infection, herpes zoster, gastrointestinal reaction, abnormal liver function, etc).

Data management: Two review authors will independently extract data on methods, patients, interventions, outcomes and results from the included studies, using a preformulated data collection form.

Quality assessment / Risk of bias analysis: The corresponding assessment tools were used according to the types of included studies.Disagreements will be resolved by discussion or by involving another reviewer.

Strategy of data synthesis: $R$ 4.0.2 software was used for meta-analysis, and odds ratio (or) values and $95 \%$ confidence intervals (CI) were selected as the effect size of meta-analysis for categorical data; For continuous type data, weighted mean difference (MD) or standardized mean difference (SMD) and $95 \% \mathrm{Cl}$ were used as effect measures. When the outcome measures included more than 3 interventions and above, the method of network meta-analysis was used for analysis, and the node analysis method was used to test the consistency; The decomp.design function was used to calculate the $Q$ statistic to analyze the heterogeneity of the included studies; And the p-score of each intervention in the study was calculated to rank the rank order of each intervention to get the best intervention. When the outcome measures included no more than 3 interventions, the method of meta-analysis was used for analysis.

Subgroup analysis: A priori defined subgroup analyses by dosing regimen (Drug dosing, course of treatment,) and 
year of publication were conducted. course of treatment.

Sensitivity analysis: Sensitivity analyses will be conducted to examine the overall effect size of the primary outcome measurement while temporarily removing: (1) each study individually from the meta-analysis, (2) studies with sample sizes $\leq \mathbf{2 0}$ across conditions, (3) studies with attrition rates $\geq 30 \%$ in at least one trial arm, and (4) studies in each rating category of overall risk of bias (ie, high- moderate respectively low risk of bias).

Country(ies) involved: China.

Keywords: Lupus Nephritis, Kunxian Capsule, meta-analysis, systematic review, protocol.

Dissemination plans: The results of this study will be published in peer-reviewed journals.

Contributions of each author:

Author 1 - Zifeng Li.

Email: 1002581008@qq.com

Author 2 - Yuling Fan.

Email: 958356961@qq.com

Author 3 - Yunhong Lei.

Author 4 - Xiaoqiang Hou.

Email: 18911093735@163.com

Author 5 - Caiyun Chang. 